\title{
A Blockchain and Internet of Things Based Architecture Design for Energy Transaction
}

\author{
You-jin Song ${ }^{1}$ and Jae-Kyu Lee ${ }^{2}$ \\ ${ }^{1}$ Deprtment of Information Management, Dongguk University at Gyeongju, South \\ Korea \\ ${ }^{2}$ Department of Technology Management Cooperation, Dongguk University at \\ Gyeongju, South Korea \\ ${ }^{1}$ song@dongguk.ac.kr, ${ }^{2}$ jaekyulee@dongguk.ac.kr
}

\begin{abstract}
Recently, the paradigm of the electric power industry has been digitized, focusing on renewable energy. While the transition to energyinformation and communications technologies (ICT) convergence is accelerating, there will be many changes in energy policy in the future. In particular, in terms of energy demand management, self-sustaining decentralization will be centered around the region, and the virtual power plant (in the form of a micro-grid) will be constructed on a regional basis according to the activation of distributed energy resources (DERs), including renewable energy. For this, business models such as the electricity trading brokerage market should also be considered. In this paper, we design an energy blockchain transaction structure that combines blockchain and the energy Internet of Things (IoT) in a virtual power plant environment. The system presented in this paper can use web-based software to Fog-enabled transactions with an integrated control system that requires intermediaries and DERs. In addition, it is possible to facilitate the activation of energy brokerage transactions by providing an environment for efficient transactions by collecting vast amounts of advanced metering infrastructure information on a fog server.
\end{abstract}

Keywords: Energy IoT, blockchain, smart contract, energy cloud, fog computing 


\section{Introduction}

The era of the 3rd Industrial Revolution, which is represented by Internet digitization and characterized by information and communication technology and automation, is being transformed into the 4th Industrial Revolution through a paradigm shift that breaks the boundaries between industries through intelligence and hyper-connection. Big data, the cloud, artificial intelligence (AI), mobile devices, and Internet of Things (IoT) technologies, which are the innovative technologies that triggered the fourth commercial revolution, are converging with energy technology, creating changes in the industrial structure and providing newly added value.

The traditional energy system, which has integrated and centrally supplied energy through large-scale facilities, has operated to create value through simple sales of a single energy source. However, with the development of small-sizedfacility technologies (such as energy storage devices) and the application of ICT, as the distributed power supply increases and interactive data exchange between supply and demand becomes possible, the operation method of the energy system is digitizing around renewable energy. While the transition to energy-ICT convergence is accelerating, there will be many changes in the energy transaction demand policy in the future.

In this way, with the development of technology and harmonization of the competitive market structure, the expansion of small-scale distributed power supplies and the combination of various energy services are creating a new energy operation system that creates added value. In particular, in terms of energy demand management, self-sustaining decentralization will be centered around the region, and the virtual grid-type power plant will be built on a regional basis with the activation of distributed energy resources (DERs), including renewable energy. Business models such as aggregation should also be considered.

On the other hand, in the era of the third industrial revolution (represented by Internet and digitalization and characterized by ICT and automation) is a paradigm breaking the boundaries between industries through intelligence and hyperconnection. Through this change is the era of the fourth industrial revolution. In particular, with IoT technology, physical things and virtual things that exist in the cyber environment are connected to each other through the Internet. It is the Internet infrastructure of the future that enables people to provide various services while being interlocked.

The Internet of Energy (IoE), in which these IoT concepts are implemented for energy, is a network infrastructure in which energy and data/information are integrated, allowing power generation and the energy storage capacity to be balanced with energy demand in real time. IoE can make to connect actively the advanced metering infrastructure (AMI), in the demand-response energy producer- 
consumer (prosumer), in vehicle-to-grid (V2G) power consumption and storage media, and in various distributed power and energy storage devices and network management. In addition, it is possible to actively integrate and manage distribution network management.

The IoE will provide a breakthrough methodology for energy distribution, energy storage, and the monitoring and communication of energy grids by providing a variety of information and connectivity to energy grids for buildings, automobiles, and cities. Using renewable energy, energy storage devices, smart meters, energy gateways, smart plugs, and home appliances, energy consumers and utility providers can save resources and costs and can provide new and powerful tools. Through the IoE, energy can be delivered in both directions anytime, anywhere; and energy consumption monitoring is possible at all levels, from individual devices to regional, national, and global units. This provides consumers with a reliable, flexible, efficient, and economical energy supply network so that centralized large-scale power plants and distributed small-scale renewable energy sources like solar and wind power can be used in one convergent system.

The International Renewable Energy Agency (IRENA) released its BLOCKCHAIN INNOVATION LANDSCAPE BRIEF (IRENA, 2019). Blockchain technology enables the implementation of smart contracts, a form of automated contract processing based on shared networks and trust between stakeholders, contributing to integrating a large portion of renewable energy through system automation. Smart contracts are automatically executed when certain conditions in the transactions for electricity exchange are met. Potential benefits can include reducing transaction costs, improving transparency, strengthening security, automating management systems, and expanding the use of renewable energy. The fields for the application of blockchain technology in renewable energy include power trading, power grid management and system operation, renewable energy-development financing, renewable-energy certification and management, and electric vehicles, as seen in Fig. 1. 


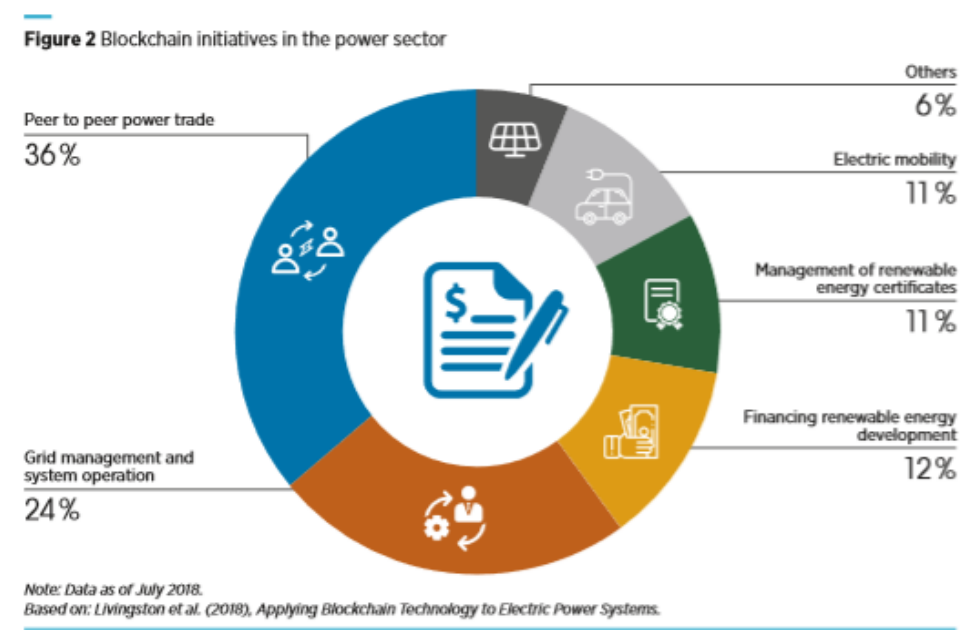

Fig. 1: Blockchain applications in renewable energy (IRENA, 2019).

It can be inefficient to add computing capabilities to all devices connected to the IoE. It is desirable to designate a somewhat suitable range of network coverage and to establish a fog (edge) computing system for a group of simple devices within range. That is, it is efficient to improve the existing gateway to a smarter base device and to maintain the simple and inexpensive low-power and low-computation specifications for the rest of the connected devices. However, if the simple sensor only transmits collected data without any self-determination, the communication costs can be very high.

Considering this point, we design an energy blockchain transaction structure that combines blockchain and the energy IoT (eIoT) in a virtual power plant (VPP) environment. When a consumer who wants to purchase energy through the smart contract function of blockchain requests a transaction from a producer-consumer (prosumer) who sells energy, the transaction information is generated in blocks to be anonymously disclosed to the participants and power companies participating in the power transaction. The transaction is verified and forms a blockchain with the contents of a previous transaction.

This paper is composed as follows. Section 1 is the introduction; Section 2 covers related research; Section 3 presents the blockchain-based fog-enabled energy IoT structure; Section 4 is our analysis and evaluation; and Section 5 is the conclusion.

\section{Related Works}

\subsection{Energy Trading}

In the energy sector, where environment-friendly, high-productivity, and stable supplies are important, demonstration projects and research and development have 
been conducted. Recently, as eco-friendly, renewable energy sources such as photovoltaic cells and wind power have been used with energy management system (EMS) technology and power conversion and energy storage system (ESS) technology, economic efficiency is increasing. Using these technologies, it is possible to increase the utilization rate by using renewable energy that is not consumed after production. To this end, research on technological measures has been conducted to expand and maximize the use and distribution of eco-friendly renewable-energy distributed resources (Young-Gon et al., 2018).

However, power consumed directly, and surplus power that is produced by selfpowered solar power generation, are constantly increasing. On the other hand, the amount of surplus power generation is not compensated for properly, which leads to a problem of lower profitability. In order to solve this challenge, trust between traders should be guaranteed without third-party intervention. In other words, it is possible to maximize utilization by establishing trust through transparency, and by automating trust-based transactions.

To this end, it is necessary to access the Industrial IoT using IoT technology and blockchain for device control. The blockchain platform for energy trading will operate safely and efficiently through higher security and greater automation than before, leading to maximized utilization (Zhetao, 2018). In this paper, the AMI controlled using IoT technology, the safety of blockchain, and the automated energy transaction structure through smart contracts are reviewed.

\subsection{Energy Clouds and Virtual Power Plants}

\subsubsection{Energy Clouds}

Recently, in the electric power field, the centralized supply structure of large-scale power plants such as thermal power, hydro power, and nuclear power has changed over to a distributed structure. The distributed power supply is expanding due to the regulation of carbon emissions and the emergence of prosumers. In addition, the installation cost of distributed power is lower, and the energy price is lower than from existing power generation. These changes are being developed into a distributed power source by fusion with technologies such as cloud computing, with technologies such as energy storage, and from energy efficiency improvement. Demand response is developing into energy clouds that control the power grid [Fig. $2]$. 


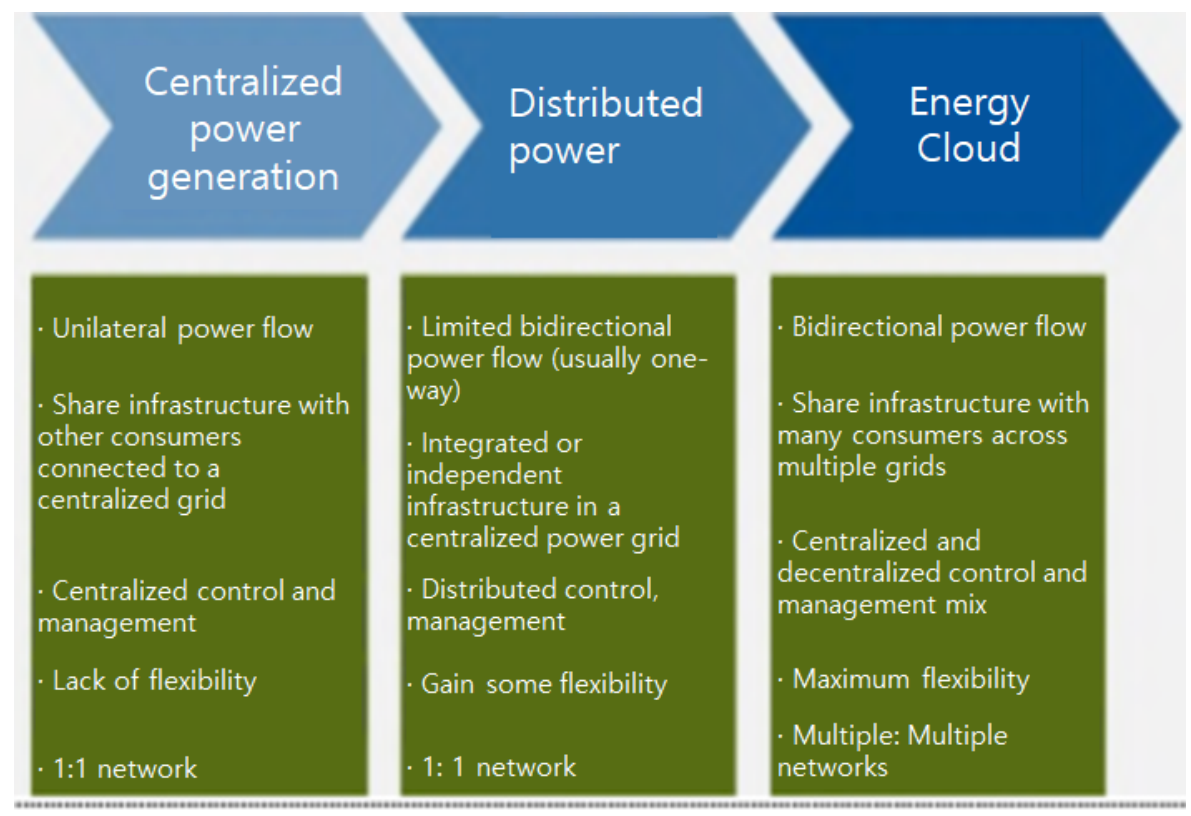

Fig. 2: The development process from centralized power generation to the energy cloud.

The energy cloud is a flexible and resilient scale economy. The decentralized power supply increases due to conversion of the energy cloud, and profits are earned by generating and selling power by itself, and promoting the growth of the smart grid market.

Energy prosumers are consumers who produce, store, and consume electric power by connecting various distributed power sources, such as photovoltaic cells, fuel cells, and the ESS. This is a concept in which a producer is fused (Giles, 2014). Furthermore, the scope can be extended to self-consumption through peer-to-peer (P2P) transactions in the community. The system for energy prosumers is a smallscale photovoltaic power generation system or a fuel cell used as the main power source, and is composed of a gateway (inverter, distribution panel, etc.) that can link to an ESS for energy storage and that can control the entire system (Giles, 2014).

\subsubsection{Virtual Power Plants}

VPPs can be used to solve or mitigate problems such as power grid congestion, peak demand, and peak hour energy costs. It is necessary to strengthen the distribution aspect before establishing a VPP from the end user side. This will be of great help in terms of better connectivity and improved reliability.

Distributed energy resources and the distributed energy resource management solution (DERMS) are key components of a VPP. The most common DER solutions are solar generation and energy storage. Currently, the most important projects related to VPPs are developed and carried out based on solar storage technology 
(Cho, 2017).

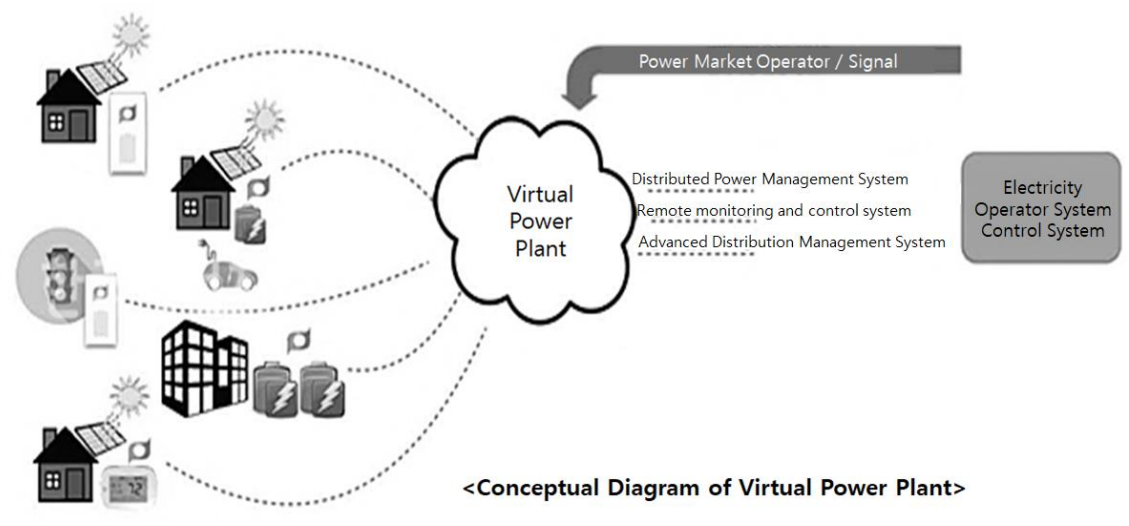

Fig. 3: Conceptual diagram of the virtual power plant (Cho, 2017).

As shown in Fig. 3, a VPP is a demand management solution that works by integrating multiple end-user power sources and connecting them to a power network. This solution will help to meet demand targets by using power generated by the building to satisfy peak building demand during peak load times. This demand management pattern can extend not only to buildings, but also to communities.

\subsection{Blockchain}

\subsubsection{Blockchain Overview}

Blockchain technology became known to the public in 2008 when Satoshi Nakamoto unveiled a study called Bitcoin: A Peer-to-Peer Electronic Cash. Based on this paper, Bitcoin has been used since the first block was created in January 2009. Blockchain deviates from the existing trading methods that depend on intermediaries, and guarantees reliability through direct transactions between trading partners. This has been a way to improve data and transaction management costs as well as security problems in the existing centralized integrated transaction system (Yoshiharu et.al, 2017).

When a bank is involved in a transaction, the account information, remittance information, and information about the money held in the bank are stored in the bank for verification, and transactions are confirmed and traded. This transaction information is stored minimally, and is accessible to a minimum number of people. That is, only banks have this information. 


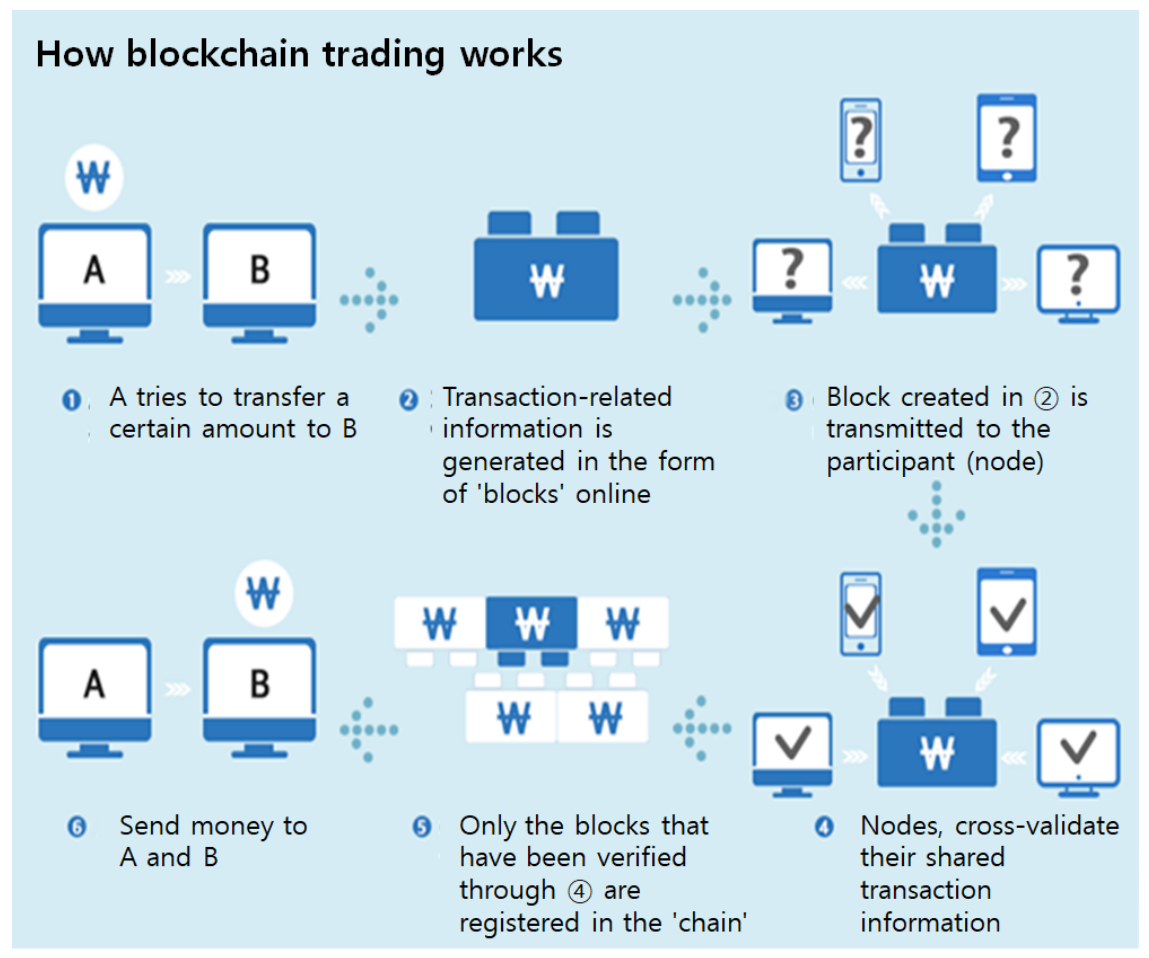

Fig. 4: Blockchain transaction order.

However, in a blockchain, first of all, the block has data information, and when users trade, it creates another block. A block is created each time a transaction is made, and these blocks are stored together with the blocks in which the existing transaction records are stored. It is called a blockchain because it is connected and stored. All transaction records stored with existing records are called public transaction books, and all information is encrypted. Then, at the time of a transaction, the PCs connected to the encrypted book are checked against each other in order to conduct the transaction. However, when doing this collation (comparing the books of all users who have a transaction book in the computer), all must agree that there is no problem in more than half of the blocks, and if so, the transaction is made. Therefore, it is difficult to counterfeit transactions because everyone has a distributed ledger [Fig. 4]. 


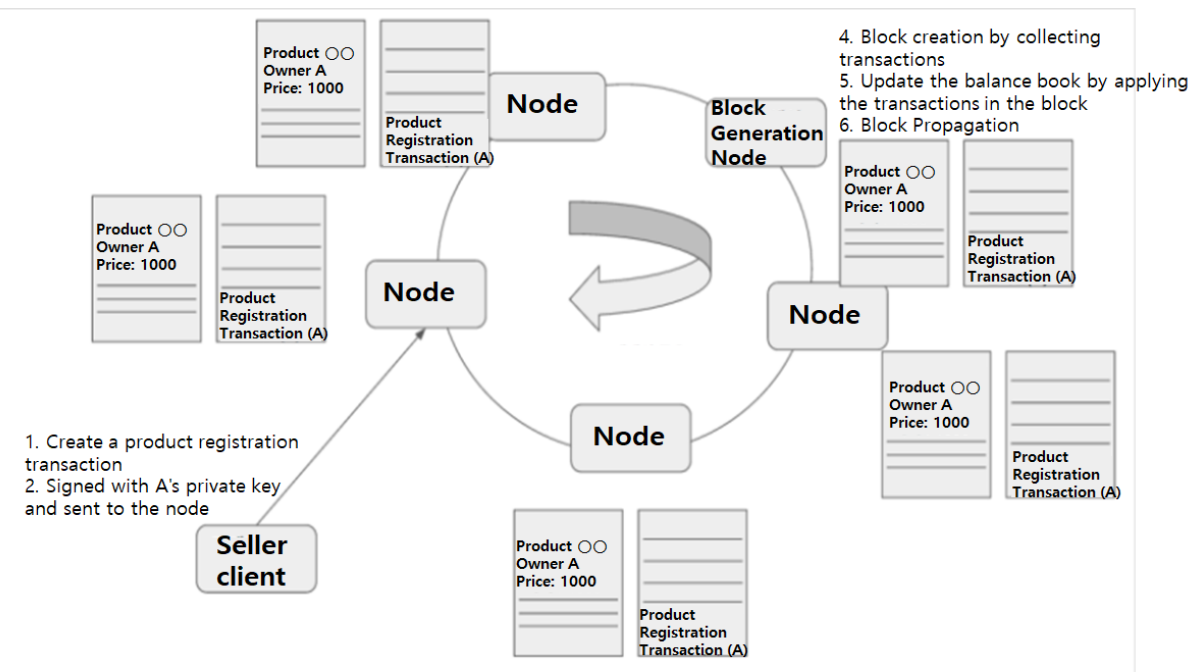

Fig. 5 Block creation and the transaction flow chart.

Blockchain has been applied in cryptocurrencies such as Bitcoin and Ethereum by using security technologies such as electronic signatures, public keys, and hash functions, and researchers are also studying how to use blockchain in financial and non-financial sectors. For Bitcoin, blockchain is a kind of decentralized digital ledger that stores the history of the movement of the value of the Bitcoin (a currency that is periodically issued). This ledger is created with cryptographic technology to prevent forge/falsification, and is used as a verification step to prevent forge/falsification of transactions through the transaction process, and to create hash values for transfers of ownership. In order to determine forgery/falsification, transaction information is collected to create a block hash, and the hash value of the previous block is also included to make a hash of the block. These blocks are connected like a chain; hence, a blockchain. Users who find a block that meets the conditions receive the value of a new Bitcoin. The process of verifying and receiving the value of the Bitcoin is called mining.

With the blockchain, there is a smart contract function on the consortium blockchain (that is, the Ethereum base). The consortium blockchain participates with authorized users and uses a method agreed to between participants. Therefore, network expansion and service speed are relatively fast, and there is little risk of attack.

\subsubsection{Smart Contract}

The smart contract is a concept first proposed by Nick Szabo in 1994 (Tokenpost, 2017). Conventional contracts are in writing, and in order to fulfill the terms of the contract, an actual person must fulfill the contract. However, if the contract is 
created with digital commands, the contract can be executed automatically according to the conditions.

Smart contracts create contracts for trade terms between traders, monitor the status of contract fulfillment through the blockchain network, and when contract conditions are satisfied, automatically execute the contents of the contract without prompting the central system to verify whether the terms of the contract were fulfilled. Through smart contracts, consumers and suppliers can quickly trade and settle energy produced and saved, and can reduce transaction fees incurred through intermediaries.
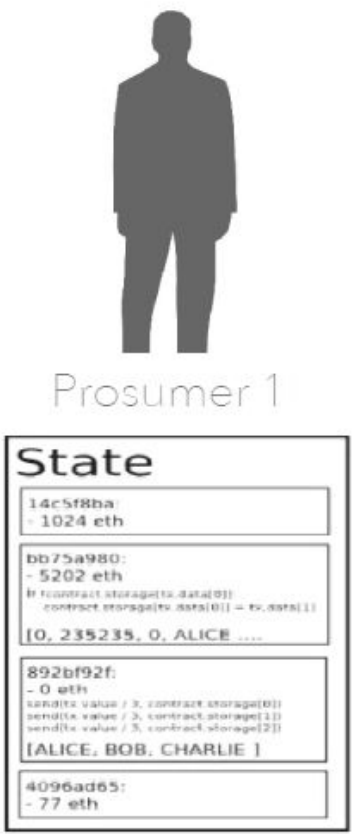
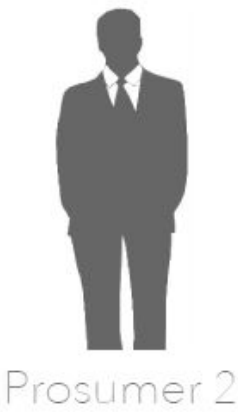

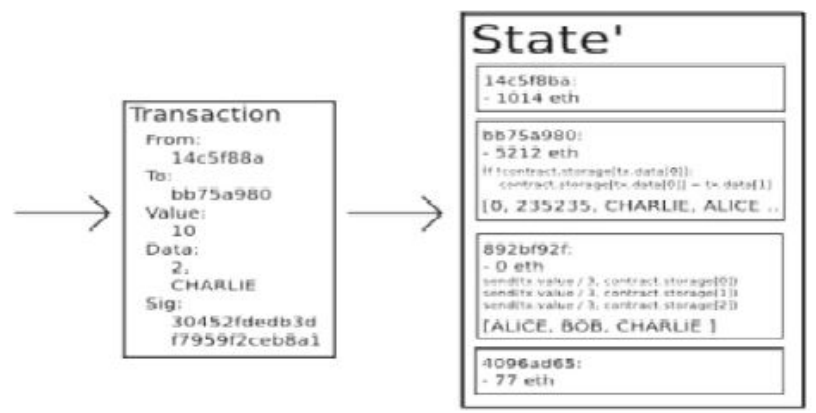

Fig. 6: The smart contract (an example).

A smart contract (shown in Figure 6) operates inside the blockchain network when a transaction occurs. All nodes in the network share transactions and store them in the transaction database (DB). The smart contract supported by blockchain executes the smart contract application according to the contents of the transaction after this step, and reflects the result in the smart contract database. For example, Prosumer 1, who acts as a supplier, creates a smart contract including various conditions, such as the amount of power to be transmitted, the payment to be transferred, and the conditions of transmission, before transmitting power to Prosumer 2, who has the demand. 


\section{Blockchain-based Fog-enabled Energy IoT}

\subsection{Overview}

The proposed blockchain-based fog-enabled eIoT system proposed in this paper is shown in Fig 7. The overall structure follows the network structure of fog computing.

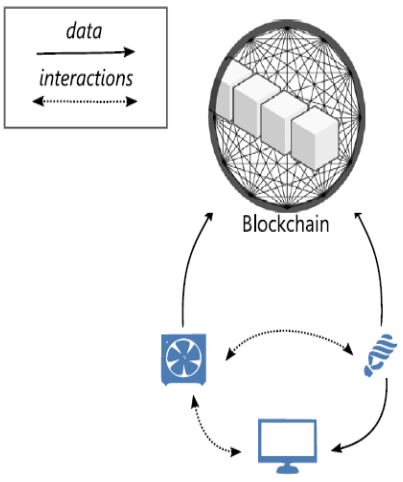

(a) IoT-IoT

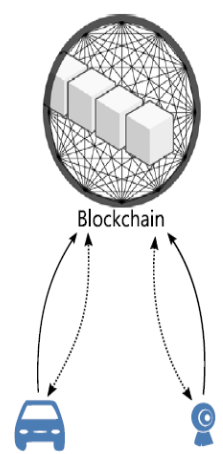

(b) IoT-Blockchain

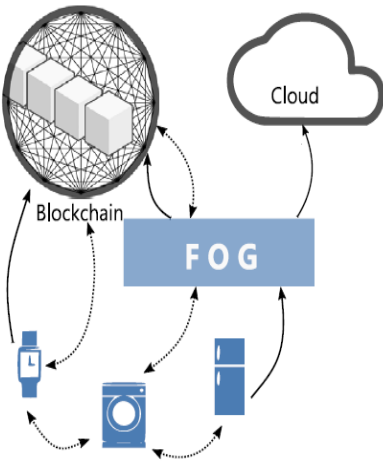

(c) Hybrid approach.

Fig. 7: The fog computing network structure (Reyna et al., 2018).

The system is divided into users, fog servers, cloud servers, and secondary users. Users create data on devices such as sensors. These data contain sensitive personal information and are stored encrypted (e.g. with attribute-based cryptography). The user can also delegate access to the encrypted data, and the secondary user has decoding authority to access the user's information, in other words, becoming a delegated authority.

A fog server collects and processes data transmitted from a sensor (for example, a smart meter for power). In addition, depending on the situation, re-encryption or monitoring can be performed first with the collected data. Fog servers are configured to be connected to each other in a P2P manner. They are authenticated, and share data with each other through the blockchain. The cloud server monitors the users' information. That is, the data received from the fog server is collected and analyzed, and the optimal decision is made based on the analyzed results.

\subsection{System Structure}

The blockchain-based energy trading platform is a platform that brokers participation in the power brokerage market and will be used for energy trading. It is a technical tool to help brokers and participants secure profit, and the main components are shown in Figure 8. It is largely divided into the service system, the VPP platform, and the AMI (Young-Gon et al., 2018). 


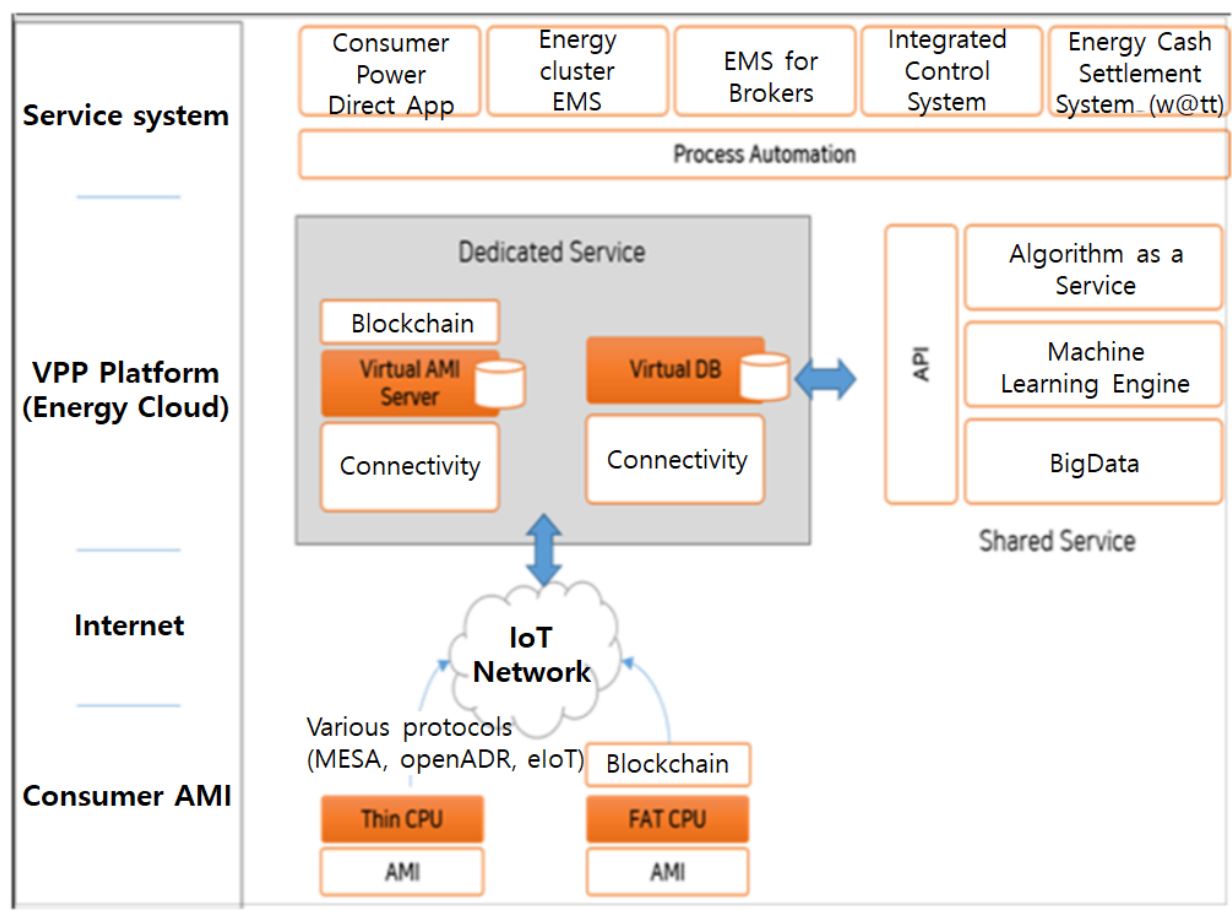

Fig. 8: The blockchain-based energy trading platform (Young-Gon et al., 2018).

The service system is provided through web-based software so that brokers and participants can easily access it. The service system consists of a consumer directtransaction app in charge of direct sales of the production of power in an energy cluster, the integrated control system required to manage the collective resources (such as an EMS in energy cluster units), and a direct power transaction cash system that settles profits through transactions. The VPP platform manages the blockchainbased energy transaction ledger, and is a core part of the system composed of machine learning and AI engines, big data analysis engines, application programming interface (API) engines, and virtual AMI servers. It can be used through the API engine in the upper layer service system. Lastly, the customer AMI enables a large amount of AMI metering information to be collected on the VPP platform's big data server through the IoT network. This can be the basis for secure transactions through authentication of power generation (reduction).

In this paper, we propose a fog-enabled energy IoT structure based on a blockchain energy trading platform (Young-Gon et al., 2018). Figure 9 shows the structure of the fog-enabled eIoT, which is largely divided into users who generate data, fog computing and the blockchain that controls energy transactions, and energy clouds that analyze and process all transaction data. Systems such as the one in Figure 8 send all of the redundant data that are not needed for analysis. This is 
not an efficient way to use a communication network to transmit data, and requires an unnecessary computation process in the energy cloud. In Figure 9, the efficiency of the system is also considered, and fog servers are linked to the energy cloud.

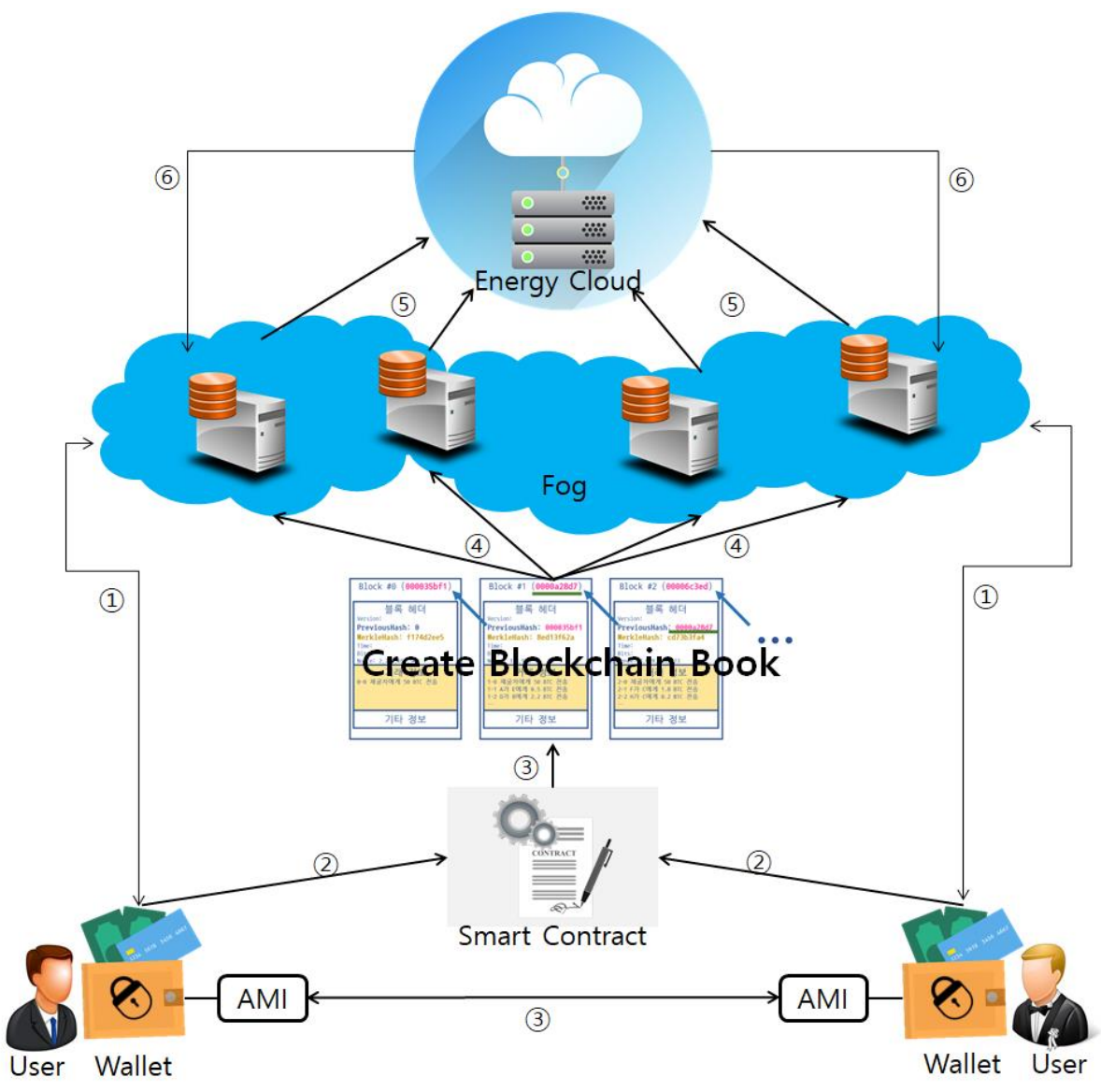

Fig. 9: The fog-enabled energy IoT structure.

The flow of the system structure is as follows.

(1) Each user receives data access through the fog servers with an account (wallet). In receiving data access authority, the system will give to the user the threshold value of the smart contract for executing the smart contract.

(2) The smart contract operates using the threshold value.

(3) As the smart contract executes, the AMI makes power transactions and block transactions for the transaction contents in order to generate the ledger.

(4) The generated books are stored in each fog server, which also store an activity log in a buffer.

(5) Only data that need to be analyzed, such as abnormal log data, from among the activity $\log$ s stored in the fog servers are transferred to the energy cloud for 
storage, analysis, and processing.

(6) The energy cloud complements and strengthens the system by updating the fog servers using the analyzed and processed data.

Through the system structure and flow suggested in this paper, it is possible to increase safety by controlling access, and it is expected to improve the system activation by improving the reliability of the system through safety. In addition, before all log data are analyzed in the cloud, it is possible to efficiently analyze the data in the fog servers by providing to the cloud only the data that need to be analyzed.

\subsection{Internal Process}

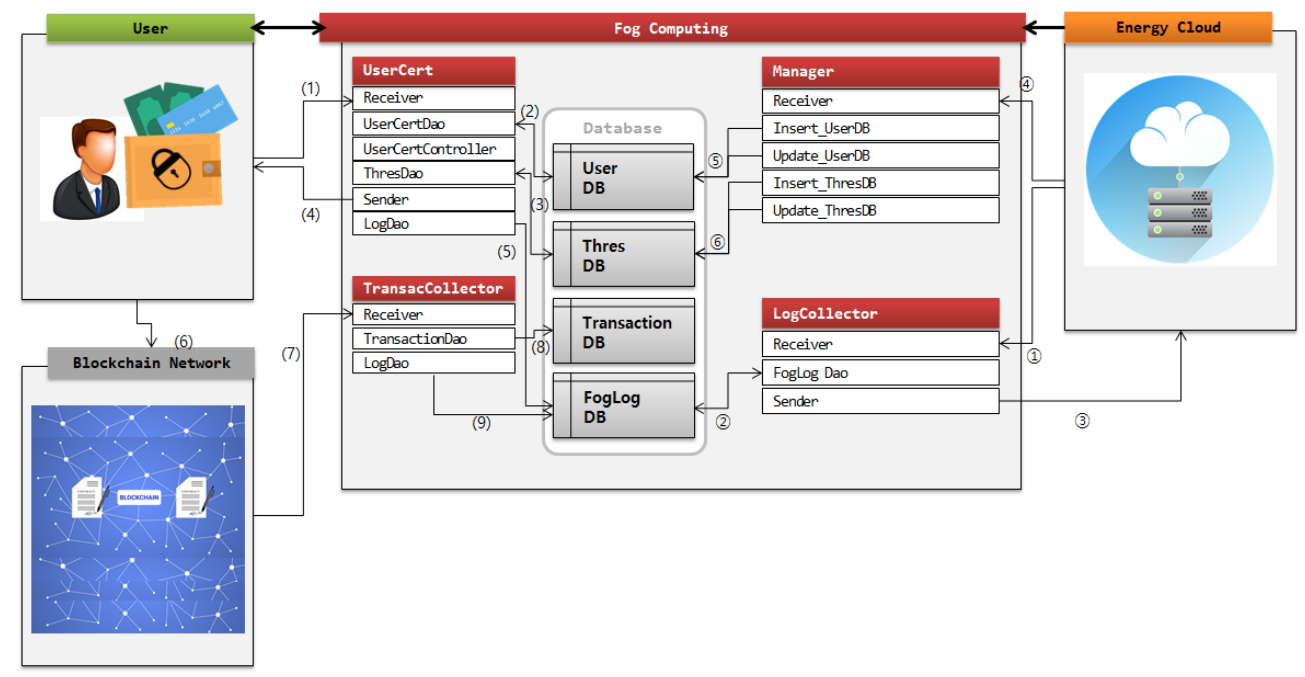

Fig. 10: The internal process of the fog-enabled eIoT system.

The internal structure of the system presented in this paper is shown in Figure 10. The system is divided into the user, the blockchain network, fog computing, and the energy cloud. The data flow inside the system is as follows.

(1) The user requests access authentication using the UserCert receiver and the desired smart contract threshold. (2) The access information requested by UserCertDao is authenticated using the user DB, and UserCertController determines whether to authenticate. Once authenticated, (3) ThresDao is used to find the threshold value desired by the accessor in ThresDB. (4) The threshold value is transferred to the user using Sender, and (5) the activity log data are stored in the FogLog DB using LogDao. (6) The user trades energy with a smart contract using the received threshold. The executed contract creates a transaction, and (7) fog computing receives the book through TransacCollector. (8) The book is stored in the Transaction DB through TransactionDao. (9) All active log data are stored in the 
FogLog DB through LogDao.

(1) The energy cloud requests log data from fog computing to be sent to LogCollector. (2) FogLog Dao retrieves all data in the FogLog DB and (3) sends it to the energy cloud via Sender. The energy cloud receives, processes, and analyzes the data. (4) The energy cloud delivers the analyzed data to Manager. (5) Manager adds and updates User DB via Insert_UserDB and Update_UserDB. (6) Threshold data are also added and updated in the Thres DB using Insert_ThresDB and Update_ThresDB.

\section{Analysis}

\subsection{Efficiency based on Fog Computing}

The existing IoT has limitations, such as processing speed and network delay, because it provides an efficient and safe energy transaction service in a hyperconnected society due to the centralized cloud form. To this end, by adding the fog layer, it is possible to overcome the limitations of the computing power and storage space in IoT devices. Traffic and latency can be efficiently managed by analyzing and storing data before it is transferred from the fog layer to the energy cloud. In addition, computing by all devices connected to one server may be inefficient. It is efficient to build one fog computing system for a group of simple devices belonging to a range of networks.

Rather than the inefficient way to raise the specifications of all devices for the hyper-connected environment, the introduction of the fog computing layer maintains the specifications of simple low-power and low-computation devices and replaces the processing of devices belonging to a certain range of networks from a specific base. This efficiently reduces the cost of establishing a connection environment.

In addition, the communication cost is efficient because the simple sensor does not indiscriminately transmit the collected data to a single server, but sends meaningful data separate from the fog layer. Significant data collected from the fog layer (e.g. generated books and activity $\operatorname{logs}$ ) are stored in each fog server, and all data are stored in the energy cloud for analysis and processing. In this way, it is possible to infer the user's situation by understanding the needs of the user through an n-th order combination and processing analysis of a variety of data, expanding it into a potential business.

\subsection{Transparency and Safety of Transaction Data}

Data and energy transaction histories through the blockchain can satisfy security and integrity. In particular, the fog-enabled blockchain is used to avoid the risk of focusing on sensitive servers, such as AMI data in cloud servers.

The public transaction ledger, where all information is encrypted, has a distributed ledger for everyone, so it is impossible to falsify the transaction history, 
and that helps secure the transactions. The user's privacy is protected through the structure for operating the smart contract after the user has been granted data access through the fog layer with an account (wallet).

Table 1: Comparative analysis

\begin{tabular}{|c|c|c|c|}
\hline Division & $\begin{array}{c}\text { IoT ( Balaji \& } \\
\text { Dakshayini, 2018) }\end{array}$ & $\begin{array}{c}\text { BIoT (Bahga \& } \\
\text { Madisetti, 2016)) }\end{array}$ & This paper \\
\hline Transparency & $\circ$ & $\circ$ & $\circ$ \\
\hline Safety & $\times$ & $\circ$ & 0 \\
\hline Efficiency & $\times$ & $\times$ & $\circ$ \\
\hline Fog-Enabled & $\times$ & $\times$ & 0 \\
\hline
\end{tabular}

$\circ$ : supported

$x$ : not supported

This paper and others (Balaji \& Dakshayini, 2018; Bahga \& Madisetti, 2016) were compared and analyzed in terms of transparency, safety, efficiency, and interlocking with the fog layer [Table 1]. An existing centralized IoT system (Balaji \& Dakshayini, 2018), another system that combines the IoT with a blockchain (Bahga \& Madisetti, 2016), and this paper all satisfy transparency. In terms of safety, due to its centralized nature, if one centralized server (Balaji \& Dakshayini, 2018 ) is exposed to an attack, all data may be lost or forged. The system (Bahga \& Madisetti, 2016) and the one in this paper use blockchain, so all distributed ledgers are held by the participants in order to satisfy data safety. In a hyper-connected environment, a structure in which traffic is concentrated in one cloud, such as the papers showed (Balaji \& Dakshayini, 2018; Bahga \& Madisetti, 2016), is inefficient. The system presented in this paper is efficient by distributing traffic through the added fog layer and reducing unnecessary traffic. In addition, this paper collects log data from the fog layer before sending data to the cloud, and collects and transmits only the data that need to be analyzed, thereby eliminating unnecessary computational processes in the cloud and increasing the efficiency of the system.

\section{Conclusion}

In this paper, we designed an energy blockchain-transaction structure that combines blockchain and the energy IoT. The structure of the system combines fog computing with an energy blockchain to escape the limitations of the IoT's processing speed and storage space. In addition, to secure and automate energy transactions, smart contracts are used in a blockchain for the fog layer. The proposed system stores log activities that control users in the fog layer between users. The fog layer not only controls the user's transactions, but also records and logs activity in the energy cloud for analysis. The energy cloud that receives the log data can analyze and process them for expansion into a potential business model. 
The system proposed in this paper will control how the user will access the system, but there is a limitation in that exposure to transaction records cannot be protected from others. In the future, we plan to strengthen security by controlling real-time access and the transaction behavior of users using AI in the fog layer.

\section{Acknowledgment}

This research was supported by the Basic Science Research Program through the National Research Foundation of Korea (NRF) funded by the Ministry of Education (2019R1F1A1056507).

\section{References}

Ah, K. S., Jong-Il, P.(2016). Energy prosumer business, new opportunities for energy business. LG Institute of economy.

A.Yoshiharu et.al. (2017). Blockchain: Architecture and Theory. Wikibooks.

Bahga, A. and Madisetti, V. (2016) Blockchain Platform for Industrial Internet of Things. Journal of Software Engineering and Applications, 9, 533-546.

Balaji, P.B.V., Dakshayini, M. (2018). An efficient data analytics-cloud integrated decision support service for smart agriculture. International Journal of Internet of Things and Big Data. 3(2),1-12.

Cho Kang Hee. (2017). Power Markets Behind Meter 'Virtual Power Generating Station'. http://www.koenergy.co.kr/news/articleView.html?idxno=90396.

Chun-Cheng Lin, Der-Jiunn Deng, Chih-Chi Kuo, \& Yu-Lin Liang. (2018). Optimal charging control of energy storage and electric vehicle of an individual in the internet of energy with energy trading. IEEE Transactions on Industrial Informatics, 14(6), 2570-2578.

David, R.K., Han, M.S. and Xuejun, L. (2017). Accurate methodology for largescale storage systems. International Journal of Internet of Things and Big Data, 2(1).

Dong-Hyun, Y. \& D. Sai Teja, R. (2016). Cloud Computing based Health Information Exchange using CDA Generation and Integration. Asia-pacific Journal of Convergent Research Interchange, 2(4), 61-68.

Energy economy institute. (2017). Development of solar PV model for households in the vicinity of nuclear power plant, global nuclear power plant market Insight.

Gai, K. , Wu, Y. , Zhu, L. , Qiu, M. , \& Shen, M. . (2019). Privacy-preserving energy trading using consortium blockchain in smart grid. IEEE Transactions on Industrial Informatics, 1-1. 
Giles Parkinson. (2014). Regulator says Australia facing 'prosumer' energy revolution. https://reneweconomy.com.au/regulator-says-australia-facing-prosumerenergy-revolution-17341/.

Hou, W. , Guo, L. , \& Ning, Z. . (2019). Local electricity storage for blockchainbased energy trading in industrial internet of things. IEEE Transactions on Industrial Informatics, 1-1.

Information and Communication Technology Promotion Center. (2017). Trend of energy research technology on the IoT. Weekly Technology Trends, 1805.

IRENA. (2019). Innovation landscape brief: Blockchain. International Renewable Energy Agency, 142.

Jindal, A., Aujla, G. S., \& Kumar, N., (2019). Survivor: a blockchain based edgeas-a-service framework for secure energy trading in sdn-enabled vehicle-to-grid environment. Computer Networks.

$\mathrm{Ju}, \mathrm{Ch}$. N. Quality of service in meta cloud. Asia-pacific Journal of Convergent Research Interchange, 1(3), 53-57.

KEDDI. (2017). Analyzing block chain concepts and use cases. KEDDI Power Economy REVIEW Unit 7.

KEMRI. (2017). Concept and application case analysis of power domain digitization. KEMRI Electric Power Economy REVIEW, 19.

Korea Energy Corporation. (2016). I want to know about the mysterious island 'Energy of Ulleung Island'. http://blog.energy.or.kr/?p=10293.

Kvaternik, K., Laszka, A., Walker, M., Schmidt, D., Sturm, M., \& Lehofer, M., et al. (2017). Privacy-preserving platform for transactive energy systems.

Li, Z., Kang, J., Yu, R., Ye, D., Deng, Q., \& Zhang, Y., (2017). Consortium blockchain for secure energy trading in industrial internet of things. IEEE Transactions on Industrial Informatics, 1-1.

Ministry of Trade. (2017). Industry and energy, plan for 3020 renewable energy transfer.

Priyamwada, Sh. (2017). A novel hybrid outlier detection scheme for health care data mining. International Journal of Internet of Things and Big Data, 2(1), 9-22.

Reyna, A. , Cristian Martín, Chen, J. , Soler, E. , \& Manuel Díaz. (2018). On blockchain and its integration with iot. challenges and opportunities. Future generation computer systems, 88(NOV.), 173-190. 
Ronnie, C. (2017). Efficient cloud data hosting availability. Asia-pacific Journal of Convergent Research Interchange, 3(2), 11-19.

Samjung KPMG Institute of economy, social city, a shared economy and civiliancentric city. (2016). Samjong INSIGHT Issue 46.

Siwoo, B. (2017). Design of efficient index management for column-based big databases. International Journal of Internet of Things and Big Data, 2(1),23-28.

SPRI. (2017). Blockchain Technology: Prospect and Implications in Perspective of Industry and Society.

Sytoogenga. (2017). Thinking about the land sale by smart contracts. Substantial Research on Land.

Tae-Yong,K. \& Hoon-Jae, L. (2017). A differential power analysis to smart doorlock system. International Journal of Mobile Device Engineering, 1(1),1-6.

Tim Weingartner. Tokenization of physical assets and the impact of IoT and AI. EU Blockchain Observatory \& Forum.

Tokenpost. (2017). What is the Smart Contract?. https://tokenpost.kr/terms/5447.

T. Sai Raaga, S. (2016). Cost minimization for big data processing in geodistributed data centres. Asia-pacific Journal of Convergent Research Interchange, 2(4), 33-41.

Ulsan Development Institute. (2016). ICT - based energy based industrial rapid growth. The response direction of Ulsan, Issue Report, 123.

WE. PRODUCT: VPP. WE Company, https://companywe.co.kr/vpp.html

You-jin, S., Jae-Kyu, L. (2020). Blockchain-based Fog-enabled Energy IoT Architecture. International Journal of Energy, Information and Communications, 11(1), pp: 23-30,

Young-Gon,K., Keol, H., Jung-In, Ch. \& Jae-Woo-Wie. (2018). A Study on the Accounts Balancing Time of Small Distributed Power Trading Platform Using Block Chain Network. Korean Society for Energy, Journal of Energy Engineering, 27(4), 86-91.

Young, H.B., Ri,R., Yong, S.K. (2019). Proposal for light shelf system that applies biomimicry for lighting energy conservation. Asia-pacific Journal of Convergent Research Interchange, 5(2), 31-38.

17 blockchain disruptive use cases. (2016). Available online: https: //everisnext.com/2016/05/31/blockchain-disruptive-usecases/. Accessed: 2018-0201 . 